\title{
Matrix Factorizations Based on Induced Norms
}

\author{
Vartan Choulakian * \\ Université de Moncton, Canada.
}

(Received: 16 August 2015; Accepted: 13 October 2015)

\begin{abstract}
We decompose a matrix $\mathbf{Y}$ into a sum of rank one bilinear forms in a stepwise manner, by considering $\mathbf{Y}$ as a mapping from the finite dimensional space $l_{r}^{n}$ to the space $l_{p}^{m}$. We provide transition formulas, and represent them in a duality diagram, thus generalizing the well known duality diagram in the french school of data analysis. As an application, we introduce a family of Euclidean multidimensional scaling models.
\end{abstract}

Keywords Hölder inequality, biconjugate decomposition, SVD, GSVD, induced norms, centroid decomposition, taxicab decomposition, transition formulas, duality diagram, multidimensional scaling.

\section{AMS 2010 subject classifications 62H25}

DOI: $10.19139 /$ soic.v4i1.160

\section{Introduction}

Matrix factorization, named also decomposition, in data analysis is at the core of factor analysis; and one of its principal aims, as clearly stated by Hubert et al. (2000), is to visualize geometrically the statistical association existing among the rows or the columns of the matrix. So the way that we factorize a matrix is of fundamental interest and concern in statistics. What is surprising is that the oldest method, the centroid factorization, see Burt (1917) and Thurstone (1931), has been rediscovered recently many times, see for instance proposal 1 in McCoy and Tropp (2011). Singular value decomposition (SVD) is the most used matrix decomposition method in statistics; the aim of this paper is to present in a coherent way the theory of SVD-like matrix factorizations based on subordinate or induced norms; and at the same time, review the existing literature. This presentation generalizes the SVD by embedding it in a larger family: It belongs to the class of optimal biconjugate decompositions; biconjugate decompositions are based on Wedderburn rank-one reduction theorem as described by Chu et al. (1995). Other alternative generalization of SVD, GSVD, is presented by Hubert et al. (2000), and which forms the basis of the french school of data analysis as reviewed recently by Holmes (2008) and De La Cruz and Holmes (2011). We also incorporate the GSVD in our representation.

This paper is organized as follows: Section 2 presents the preliminaries concerning induced or subordinate matrix norms; section 3 presents the matrix factorizations based on induced norms; section 4 presents an overview of the french school of data analysis, and we conclude in section 5.

\section{Preliminaries on real Banach spaces $l_{p}^{n}$}

We start with some preliminaries and at the same time introduce notation. We note: $l_{p}^{n}:=\left(\mathbb{R}^{n},\|\cdot\|_{p}\right)$ is a finite dimensional Banach space; that is, $\mathbb{R}^{n}$ is $n$-dimensional complete vector space with the $p$-norm, $\|.\| \|_{p}$, for $p \geq 1$.

\footnotetext{
*Correspondence to: Vartan Choulakian (Email:vartan.choulakian@umoncton.ca.)
}

ISSN 2310-5070 (online) ISSN 2311-004X (print)

Copyright (C) 2016 International Academic Press 
For an $\mathbf{x} \in \mathbb{R}^{n}$, its $p$-norm is defined as $\|\mathbf{x}\|_{1}=\sum_{i=1}^{n}\left|x_{i}\right|$ for $p=1,\|\mathbf{x}\|_{p}=\left(\sum_{i=1}^{n}\left|x_{i}\right|^{p}\right)^{1 / p}$ for $p>1$, and $\|\mathbf{x}\|_{\infty}=\max _{i=1}^{n}\left|x_{i}\right|$ for $p=\infty$.

The norm $\|\mathbf{x}\|_{p}$ has the following four properties

(N1) $\|\mathbf{x}\|_{p} \geq 0$

(N2) $\|\mathbf{x}\|_{p}=0$ iff $\mathbf{x}=\mathbf{0}$

(N3) $\|\alpha \mathbf{x}\|_{p}=|\alpha|\|\mathbf{x}\|_{p}$ for $\alpha \in \mathbb{R}$

(N4) $\|\mathbf{x}+\mathbf{y}\|_{p} \leq\|\mathbf{x}\|_{p}+\|\mathbf{y}\|_{p}$

(N4) implies: || $\mathbf{x}\left\|_{p}-\right\| \mathbf{y}\left\|_{p} \mid \leq\right\| \mathbf{x}-\mathbf{y} \|_{p}$, from which we deduce that the $p$-norm is a continuous mapping of $\mathbb{R}^{n}$ into $\mathbb{R}$.

The proof of (N4) is based on Hölder and Minkowski inequalities.

We define the unit sphere to be

$$
S_{p}^{n}=\left\{\mathbf{x} \in \mathbb{R}^{n}:\|\mathbf{x}\|_{p}=1\right\},
$$

and $\left(p, p_{1}\right)$ designate the conjugate pair, that is, $\frac{1}{p}+\frac{1}{p_{1}}=1$ for $p \geq 1$ and $p_{1} \geq 1$.

\section{Hölder inequality:}

$$
<\mathbf{x}^{*}, \mathbf{x}>\leq\left\|\mathbf{x}^{*}\right\|_{p_{1}}\|\mathbf{x}\|_{p} \text { for } \mathbf{x}^{*} \in l_{p_{1}}^{n} \text { and } \mathbf{x} \in l_{p}^{n}
$$

or

$$
<\mathbf{x}^{*}, \mathbf{x}>\leq\|\mathbf{x}\|_{p} \text { for } \mathbf{x}^{*} \in S_{p_{1}}^{n} \text { and } \mathbf{x} \in l_{p}^{n}
$$

or

$$
<\mathrm{x}^{*}, \mathrm{x}>\leq 1 \text { for } \mathrm{x}^{*} \in S_{p_{1}}^{n} \text { and } \mathrm{x} \in S_{p}^{n} .
$$

Note that $\left\langle\mathbf{x}^{*}, \mathbf{x}\right\rangle=\sum_{i=1}^{n} x_{i}^{*} x_{i}=\left(\mathbf{x}^{*}\right)^{\prime} \mathbf{x}=\mathbf{x}^{\prime} \mathbf{x}^{*}$, where $\mathbf{x}^{\prime}$ is the transpose of the row vector $\mathbf{x}$; further, $<\mathbf{x}^{*}, \mathbf{x}>$ represents a scalar product only when the conjugate pair $\left(p, p_{1}\right)=(2,2)$. The next result is an application of Hölder inequality.

Lemma 1: Let $\mathbf{x} \in l_{p}^{n}$, then there exists a norming functional $\varphi(\mathbf{x}) \in S_{p_{1}}^{n}$ such that $\langle\varphi(\mathbf{x}), \mathbf{x}\rangle=\|\mathbf{x}\|_{p}=$ $\max <\mathbf{x}^{*}, \mathbf{x}>$ subject to $\mathbf{x}^{*} \in S_{p_{1}}^{n}$.

Proof: Explicitly we have:

$$
\begin{aligned}
\varphi(\mathbf{x}) & =\left(v_{j}=\operatorname{sgn}\left(x_{j}\right)\right) \quad \text { for } p=1 \\
& =\left(v_{j}=\operatorname{sgn}\left(x_{j}\right)\left|\frac{x_{j}}{\|\mathbf{x}\|_{p}}\right|^{p-1}\right) \quad \text { for } p>1 \\
& =\mathbf{e}_{\alpha} \operatorname{sgn}\left(x_{\alpha}\right) \quad \text { for } p=\infty,
\end{aligned}
$$

where $\left\{\mathbf{e}_{\beta}: \beta=1, \ldots, n\right\}$ designates the canonical basis and $x_{\alpha}=\arg \max _{\beta=1}^{n}\left|x_{\beta}\right|$. It is easy to show that $\varphi(\mathbf{x}) \in S_{p_{1}}^{n}$ for any $\mathbf{x} \in l_{p}^{n}$ and $\mathbf{x} \neq \mathbf{0}$; that is, $\|\varphi(\mathbf{x})\|_{p_{1}}=1$.

Remark: In more general settings, Lemma 1 is proven as a corollary to the famous Hahn-Banach theorem, see for instance Kreyszig (1978, p.223).

Example 1: Consider the vector $\mathbf{x}^{\prime}=\left(\begin{array}{llll}1 & 2 & -1 & -2\end{array}\right)$.

a) If $\mathbf{x} \in l_{2}^{4}$, then $\|\mathbf{x}\|_{2}=10^{1 / 2}$ and $\varphi(\mathbf{x})=\frac{\mathbf{x}}{10^{1 / 2}} \in S_{2}^{4}$ and $<\varphi(\mathbf{x}), \mathbf{x}>=10^{1 / 2}$. Explicitly $\varphi(\mathbf{x})^{\prime}=\left(\begin{array}{lll}1 & 2\end{array}\right.$ $1-2) / 10^{1 / 2}$.

b) If $\mathbf{x} \in l_{1}^{4}$, then $\|\mathbf{x}\|_{1}=6$ and $\varphi(\mathbf{x})=\operatorname{sgn}(\mathbf{x}) \in S_{\infty}^{4}$ and $<\varphi(\mathbf{x}), \mathbf{x}>=6$. Explicitly $\varphi(\mathbf{x})^{\prime}=\left(\begin{array}{llll}1 & 1 & -1\end{array}\right.$ 1).

c) If $\mathbf{x} \in l_{\infty}^{4}$, then $\|\mathbf{x}\|_{\infty}=2$ and $\varphi(\mathbf{x})=-\mathbf{e}_{4} \in S_{1}^{4}$ and $<\varphi(\mathbf{x}), \mathbf{x}>=2$. Explicitly $\varphi(\mathbf{x})^{\prime}=\left(\begin{array}{llll}0 & 0 & 0 & -1\end{array}\right)$. Another value is: $\varphi(\mathbf{x})^{\prime}=\left(\begin{array}{llll}0 & 1 & 0 & 0\end{array}\right)$. 
d) If $\mathbf{x} \in l_{3}^{4}$, then $\|\mathbf{x}\|_{3}=18^{1 / 3}$ and $\varphi(\mathbf{x})=\left(v_{j}=\frac{x_{j}^{2}}{18^{2 / 3}} \operatorname{sgn}\left(x_{j}\right)\right) \in S_{1.5}^{4}$ and $<\varphi(\mathbf{x}), \mathbf{x}>=18^{1 / 3}$. Explicitly $\varphi(\mathbf{x})^{\prime}=\left(\begin{array}{llll}1 & 4 & -1 & -4\end{array}\right) / 18^{2 / 3}$.

Let $B\left(l_{r}^{n}, l_{p}^{m}\right)$ be the set of bounded linear maps (operators) from $l_{r}^{n}$ to $l_{p}^{m}$, which we identify with the set of $m \times n$ real matrices in the usual way. A given matrix $\mathbf{A}$ can be interpreted in three different ways as an operator: $\mathbf{A} \in B\left(l_{r}^{n}, l_{p}^{m}\right)$ or $\mathbf{A}^{\prime} \in B\left(l_{p_{1}}^{m}, l_{r_{1}}^{n}\right)$ or $\mathbf{A} \in B\left(l_{r}^{n} \times{ }_{p_{1}}^{m}, \mathbb{R}\right)$. For $\mathbf{A} \in B\left(l_{r}^{n}, l_{p}^{m}\right)$ its induced or subordinate norm is defined to be

$$
\begin{aligned}
\|\mathbf{A}\|_{r \rightarrow p} & =\max \left\{\|\mathbf{A} \mathbf{u}\|_{p}: \mathbf{u} \in S_{r}^{n}\right\} \\
& =\left\|\mathbf{A} \mathbf{u}_{1}\right\|_{p} \text { for } \mathbf{u}_{1} \in S_{r}^{n} \\
& =\left\|\mathbf{a}_{1}\right\|_{p} \text { for } \mathbf{a}_{1} \in l_{p}^{m} .
\end{aligned}
$$

Similarly, for $\mathbf{A}^{\prime} \in B\left(l_{p_{1}}^{m}, l_{r_{1}}^{n}\right)$ its induced or subordinate norm is

$$
\begin{aligned}
\left\|\mathbf{A}^{\prime}\right\|_{p_{1} \rightarrow r_{1}} & =\max \left\{\left\|\mathbf{A}^{\prime} \mathbf{v}\right\|_{r_{1}}: \mathbf{v} \in S_{p_{1}}^{m}\right\} \\
& =\left\|\mathbf{A}^{\prime} \mathbf{v}_{1}\right\|_{r_{1}} \text { for } \mathbf{v}_{1} \in S_{p_{1}}^{m} \\
& =\left\|\mathbf{b}_{1}\right\|_{r_{1}} \text { for } \mathbf{b}_{1} \in l_{r_{1}}^{n} .
\end{aligned}
$$

Finally, for $\mathbf{A} \in B\left(l_{r}^{n} \times{ }_{p_{1}}^{m}, \mathbb{R}\right)$ as a bilinear functional its induced or subordinate norm is

$$
\lambda_{1}=\max \left\{\mathbf{v}^{\prime} \mathbf{A u}: \mathbf{u} \in S_{r}^{n} \text { and } \mathbf{v} \in S_{p_{1}}^{m}\right\} .
$$

In (1, 2 and 3), the maximum values are attained; for further details see for instance Kreyszig (1978, section 2.5). Essentially, we are computing the quintuplet $\left(\mathbf{a}_{1}, \mathbf{b}_{1}, \mathbf{u}_{1}, \mathbf{v}_{1}, \lambda_{1}\right)$, and the next theorem is a central result which shows the relations among the elements of the quintuplet.

\section{Theorem 1:}

$$
\begin{aligned}
\|\mathbf{A}\|_{r \rightarrow p} & =\left\|\mathbf{A}^{\prime}\right\|_{p_{1} \rightarrow r_{1}}=\lambda_{1} \\
& =\mathbf{v}_{1}^{\prime} \mathbf{a}_{1}=\mathbf{b}_{1}^{\prime} \mathbf{u}_{1},
\end{aligned}
$$

where

$$
\mathbf{A u}_{1}=\mathbf{a}_{1} \text { and } \mathbf{v}_{1}=\varphi\left(\mathbf{a}_{1}\right)
$$

and

$$
\mathbf{A}^{\prime} \mathbf{v}_{1}=\mathbf{b}_{1} \text { and } \mathbf{u}_{1}=\varphi\left(\mathbf{b}_{1}\right)
$$

the last two equations are known as transition formulas.

Proof: For $\mathbf{u} \in S_{r}^{n}$ and $\mathbf{v} \in S_{p_{1}}^{m}$, we consider the bilinear form

$$
\begin{aligned}
\lambda(\mathbf{u}, \mathbf{v}) & =\mathbf{v}^{\prime} \mathbf{A} \mathbf{u} \\
& \leq\|\mathbf{A} \mathbf{u}\|_{p} \text { by Hölder inequality for } \mathbf{A} \mathbf{u} \in l_{p}^{n} \\
& \leq \max _{\mathbf{u} \in S_{r}^{n}}\|\mathbf{A} \mathbf{u}\|_{p}=\|\mathbf{A}\|_{r \rightarrow p} \text { by }(1) \\
& =\left\|\mathbf{A} \mathbf{u}_{1}\right\|_{p}=\mathbf{v}_{1}^{\prime} \mathbf{A} \mathbf{u}_{1} \text { where } \mathbf{v}_{1}=\varphi\left(\mathbf{A} \mathbf{u}_{1}\right)=\varphi\left(\mathbf{a}_{1}\right) \\
& =\max _{\mathbf{v} \in S_{p_{1}}^{m}} \mathbf{v}^{\prime} \mathbf{A} \mathbf{u}_{1} \text { by Lemma } 1 \\
& =\max _{\mathbf{v} \in S_{p_{1}}^{m}} \max _{\mathbf{u} \in S_{r}^{n}} \mathbf{v}^{\prime} \mathbf{A} \mathbf{u} .
\end{aligned}
$$


Now using (6 and 7) and replacing $\mathbf{A}$ by $\mathbf{A}^{\prime}$ we have

$$
\begin{aligned}
\|\mathbf{A}\|_{r \rightarrow p} & =\mathbf{v}_{1}^{\prime} \mathbf{A} \mathbf{u}_{1} \\
& =\mathbf{u}_{1}^{\prime} \mathbf{A}^{\prime} \mathbf{v}_{1} \text { where } \mathbf{u}_{1}=\varphi\left(\mathbf{A}^{\prime} \mathbf{v}_{1}\right)=\varphi\left(\mathbf{b}_{1}\right) \\
& =\left\|\mathbf{A}^{\prime}\right\|_{p_{1} \rightarrow r_{1}}
\end{aligned}
$$

which is the required result.

The transition formulas (4 and 5) can be represented by the following duality diagram

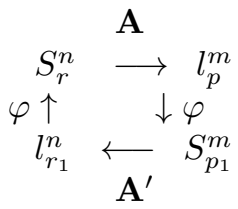

\section{Remark 1:}

a) The geometrical-statistical interpretation of Theorem 1 is that $\lambda_{1}$ is the largest dispersion value by which the operator $\mathbf{A}$ stretches an element of $\mathbf{u} \in S_{r}^{n} ; \mathbf{u}_{1}$ is called the first principal axis of the rows of $\mathbf{A}$, and $\mathbf{a}_{1}$ represents the projected values of the rows of $\mathbf{A}$ on $\mathbf{u}_{1}$, and we name it the first projected row factor or the first principal component. And by duality, we also have $\lambda_{1}$ is the largest dispersion value by which the operator $\mathbf{A}^{\prime}$ stretches an element of $\mathbf{v} \in S_{p_{1}}^{m} ; \mathbf{v}_{1}$ is called the first principal axis of the columns of $\mathbf{A}$, and $\mathbf{b}_{1}$ is the first column projected factor which represents the projected values of the columns of $\mathbf{A}$ on $\mathbf{v}_{1}$.

b) The vectors $\mathbf{a}_{1}, \mathbf{b}_{1}, \mathbf{u}_{1}$ and $\mathbf{v}_{1}$ belong to four different spaces: $\mathbf{a}_{1} \in l_{p}^{m}, \quad \mathbf{b}_{1} \in l_{r_{1}}^{n}, \mathbf{u}_{1} \in S_{r}^{n} \subset l_{r}^{n} \quad$ and $\mathbf{v}_{1} \in S_{p_{1}}^{m} \subset l_{p_{1}}^{m}$.

c) The transition formulas provide us an iterative algorithm to compute a maximum of $\left\{\|\mathbf{A u}\|_{p}: \mathbf{u} \in S_{r}^{n}\right\}$; this maximum value can be a relative maximum. The norm $\|\mathbf{A}\|_{r \rightarrow p}$ corresponds to the absolute maximum. The algorithm is named the power method for $l_{p}$ norm by Boyd (1974); Wold's (1966) NIPALS (nonlinear iterative partial alternating least squares) algorithm, named also criss-cross regression by Gabriel and Zamir (1979), is a particular case. The algorithm can be summarized in the following way, where $\mathbf{b}$ is a starting value:

Step 1: $\mathbf{u}=\varphi(\mathbf{b}), \mathbf{a}=\mathbf{A u}$ and $\lambda(\mathbf{a})=\|\mathbf{a}\|_{p} ;$

Step 2: $\mathbf{v}=\varphi(\mathbf{a}), \mathbf{b}=\mathbf{A}^{\prime} \mathbf{v}$ and $\lambda(\mathbf{b})=\|\mathbf{b}\|_{r_{1}}$;

Step 3: If $\lambda(\mathbf{b})-\lambda(\mathbf{a})>\epsilon$ fixed value, go to Step 1; otherwise, stop.

The proof of the convergence of the algorithm is based on application of Hölder inequality twice: Let $\mathbf{u}^{(k)}, \mathbf{v}^{(k)}$ and $\lambda^{(k)}$ for $k \geq 1$ represent the $k$ th iteration values, then:

$$
\begin{aligned}
\lambda^{(k)} & =\mathbf{v}^{(k) \prime} \mathbf{A} \mathbf{u}^{(k)} \\
& \leq\left(\mathbf{v}^{(k) \prime} \mathbf{A}\right) \varphi\left(\mathbf{A}^{\prime} \mathbf{v}^{(k)}\right) \quad \text { by Hölder inequality } \\
& =\mathbf{v}^{(k) \prime} \mathbf{A} \mathbf{u}^{(k+1)} \\
& \leq \varphi\left(\mathbf{A u} \mathbf{u}^{(k+1)}\right)^{\prime}\left(\mathbf{A} \mathbf{u}^{(k+1)}\right) \text { by Hölder inequality } \\
& =\mathbf{v}^{(k+1) \prime} \mathbf{A} \mathbf{u}^{(k+1)}=\lambda^{(k+1)} .
\end{aligned}
$$

The rows or the columns of $\mathbf{A}$ can be used as starting values for $\mathbf{a}$ or $\mathbf{b}$.

\subsection{Particular norms}

Let $\mathbf{A} \in B\left(l_{r}^{n}, l_{p}^{m}\right)$, then $\mathbf{A}^{\prime} \in B\left(l_{p_{1}}^{m}, l_{r_{1}}^{n}\right)$. In general the conjugate pairs $\left(r, r_{1}\right)$ and $\left(p_{1}, p\right)$ are not equal, which implies that the geometry of the rows is different from the geometry of the columns. If $\left(r, r_{1}\right)=\left(p_{1}, p\right)=(r, p)$, then the geometric structure defined on the rows of $\mathbf{A}$ is identical to the geometric structure defined on the columns of $\mathbf{A}$; this class is named transposition invariant by Choulakian (2005a). 
For two particular values of the conjugate pairs $\left(r, r_{1}\right)$, explicit formulas are available; however, the spectral norm is the most well known, which is transposition invariant.

Proposition 1: For $\left(r, r_{1}\right)=(\infty, 1)$, then

$$
\begin{aligned}
\|\mathbf{A}\|_{\infty \rightarrow p} & =\left\|\mathbf{A}^{\prime}\right\|_{p_{1} \rightarrow 1} \text { by Theorem } 1 \\
& =\max _{\mathbf{u}}\|\mathbf{A u}\|_{p} \text { subject to } \mathbf{u} \in\{-1,+1\}^{n} .
\end{aligned}
$$

The proof is based on Hölder inequality: For any $\mathbf{v} \in S_{p_{1}}^{m}$ consider

$$
\begin{aligned}
\left\|\mathbf{A}^{\prime} \mathbf{v}\right\|_{1} & =\mathbf{u}^{\prime} \mathbf{A}^{\prime} \mathbf{v} \text { for } \mathbf{u}=\operatorname{sgn}\left(\mathbf{A}^{\prime} \mathbf{v}\right) \in\{-1,+1\}^{n} \text { by Lemma } 1 \\
& \leq\|\mathbf{A u}\|_{p} \text { for } \mathbf{u} \in\{-1,+1\}^{n} \text { by Hölder inequality, } \\
& \leq \max \|\mathbf{A} \mathbf{u}\|_{p} \text { for } \mathbf{u} \in\{-1,+1\}^{n} \\
& =\left\|\mathbf{A} \mathbf{u}_{1}\right\|_{p} \text { where } \mathbf{u}_{1}=\arg \max \|\mathbf{A u}\|_{p} \text { subject to } \mathbf{u} \in\{-1,+1\}^{n}
\end{aligned}
$$

By Lemma 1, if $\mathbf{v}=\varphi\left(\mathbf{A u}_{1}\right)$, then $\left\|\mathbf{A}^{\prime}\right\|_{p_{1} \rightarrow 1}=\max \left\{\left\|\mathbf{A}^{\prime} \mathbf{v}\right\|_{1}: \mathbf{v} \in S_{p_{1}}^{m}\right\}=\max _{\mathbf{u}}\|\mathbf{A u}\|_{p} \quad$ subject to $\mathbf{u} \in$ $\{-1,+1\}^{n}$, which is the required result.

Proposition 2: For $\left(r, r_{1}\right)=(1, \infty)$, then

$$
\begin{aligned}
\|\mathbf{A}\|_{1 \rightarrow p} & =\left\|\mathbf{A}^{\prime}\right\|_{p_{1} \rightarrow \infty} \text { by Theorem } 1 \\
& =\max _{\alpha=1}^{m}\left\|\mathbf{A}_{* \alpha}\right\|_{p},
\end{aligned}
$$

where $\mathbf{A}_{* \alpha}$ is the $\alpha$ th column of $\mathbf{A}$.

The proof is similar to the proof in Proposition 1. For any $\mathbf{v} \in S_{p_{1}}^{m}$ consider

$$
\begin{aligned}
& \left\|\mathbf{A}^{\prime} \mathbf{v}\right\|_{\infty}=\mathbf{u}^{\prime} \mathbf{A}^{\prime} \mathbf{v} \text { for } \mathbf{u}=\mathbf{e}_{\alpha} \operatorname{sgn}\left(x_{\alpha}\right) \text { and } x_{\alpha}=\arg \max _{\beta=1}^{n}\left|\mathbf{A}^{\prime} \mathbf{v}\right| \text { by Lemma } 1, \\
& \leq\|\mathbf{A u}\|_{p} \text { for } \mathbf{u}=\mathbf{e}_{\alpha} \operatorname{sgn}\left(x_{\alpha}\right) \text { and } x_{\alpha}=\arg \max _{\beta=1}^{n}\left|\mathbf{A}^{\prime} \mathbf{v}\right| \text { by Hölder inequality, } \\
& \leq\left\|\mathbf{A} \mathbf{u}_{1}\right\|_{p} \text { for } \mathbf{u}_{1}=\arg \max _{\mathbf{u}}\left\|\mathbf{A} \mathbf{e}_{\alpha}\right\|_{p}, \\
& =\max _{\alpha=1}^{m}\left\|\mathbf{A}_{* \alpha}\right\|_{p} \text {. }
\end{aligned}
$$

By Theorem 1, if $\mathbf{v}=\varphi\left(\mathbf{A} \mathbf{u}_{1}\right)$, then $\max \left\{\left\|\mathbf{A}^{\prime} \mathbf{v}\right\|_{\infty}: \mathbf{v} \in S_{p_{1}}^{m}\right\}=\max _{\alpha=1}^{m}\left\|\mathbf{A}_{* \alpha}\right\|_{p}=\max _{\mathbf{u}}\|\mathbf{A u}\|_{p}$ subject to $\mathbf{u}=\mathbf{e}_{\alpha}$ for $\alpha=1, \ldots, n$, which is the required result.

Proposition 3: For $\left(r, r_{1}\right)=(2,2)$, then

$$
\begin{aligned}
\|\mathbf{A}\|_{2 \rightarrow 2} & =\left\|\mathbf{A}^{\prime}\right\|_{2 \rightarrow 2} \\
& =\sqrt{\lambda_{\max }\left(\mathbf{A A}^{\prime}\right)} \\
& =\sqrt{\lambda_{\max }\left(\mathbf{A}^{\prime} \mathbf{A}\right)},
\end{aligned}
$$

where $\lambda_{\max }$ is the greatest eigenvalue of $\mathbf{A} \mathbf{A}^{\prime}$ or $\mathbf{A}^{\prime} \mathbf{A}$, and it is named spectral norm.

Drakakis and Pearlmutter (2009) and Lewis (2010) discuss the following nine cases of $\|\mathbf{A}\| \|_{r \rightarrow p}$ for $r, p=1,2,3$, which can be easily deduced from the above results. 


\section{Matrix factorizations}

Let $\mathbf{X} \in B\left(l_{r}^{n}, l_{p}^{m}\right)$. Let $\left(\mathbf{a}_{1}, \mathbf{b}_{1}\right)$ be the first projected factors associated with $\lambda_{1}$. We repeat the above procedure on the residual dataset

$$
\begin{aligned}
\mathbf{X}^{(1)} & =\mathbf{X}-\mathbf{a}_{1} \mathbf{b}_{1}^{\prime} / \lambda_{1} \\
& =\left(\mathbf{I}_{m}-\mathbf{P}_{a_{1}}\right) \mathbf{X} \\
& =\mathbf{X}\left(\mathbf{I}_{n}-\mathbf{P}_{b_{1}}\right)
\end{aligned}
$$

where $\mathbf{P}_{\mathbf{a}_{1}}=\mathbf{a}_{1} \mathbf{v}_{1}^{\prime} / \lambda_{1}$ is the projection operator on $\mathbf{a}_{1} \in l_{p}^{m}$, because $\mathbf{P}_{\mathbf{a}_{1}}^{2}=\mathbf{P}_{\mathbf{a}_{1}}$. Similarly, $\mathbf{P}_{\mathbf{b}_{1}}=\mathbf{b}_{1} \mathbf{u}_{1}^{\prime} / \lambda_{1}$ is the projection operator on $\mathbf{b}_{1} \in l_{r_{1}}^{n}$. We note that the $\operatorname{rank}\left(\mathbf{X}^{(1)}\right)=\operatorname{rank}(\mathbf{X})-1$, because

$$
\mathbf{X}^{(1)} \mathbf{u}_{1}=\mathbf{0} \text { and } \mathbf{X}^{(1) \prime} \mathbf{v}_{1}=\mathbf{0}
$$

which implies that

$$
\mathbf{u}_{1}^{\prime} \mathbf{b}_{2}=0 \text { and } \mathbf{v}_{1}^{\prime} \mathbf{a}_{2}=0,
$$

where $\mathbf{b}_{2}$ and $\mathbf{a}_{2}$ are the vectors $\mathbf{b}$ and $\mathbf{a}$ relative to matrix $\mathbf{X}^{(1)}$ respectively. Equations $(8,9,10)$ are known as Wedderburn's rank one reduction formula, see Chu, Funderlic and Golub (1995). By repeating the above procedure we get the data reconstitution formula for the matrix $\mathbf{X}$ as a function of the projected row and column factor coordinates $\left(\mathbf{a}_{\alpha}, \mathbf{b}_{\alpha}\right)$ associated with the dispersion values $\lambda_{\alpha}$, for $\alpha=1, \ldots, k$, and $k=\operatorname{rank}(\mathbf{X})$,

$$
\mathbf{X}=\sum_{\alpha=1}^{\mathbf{k}} \mathbf{a}_{\alpha} \mathbf{b}_{\alpha}^{\prime} / \lambda_{\alpha}
$$

or elementwise

$$
x_{i j}=\sum_{\alpha=1}^{k} a_{\alpha}(i) b_{\alpha}(j) / \lambda_{\alpha} .
$$

Equation (11) represents the decomposition of $\mathbf{X}$ based on $l_{r}^{n} \rightarrow l_{p}^{m}$ induced norm.

\subsection{The case of $X$ symmetric}

When the matrix $\mathbf{X}$ is symmetric, we can have a symmetric decomposition or a nonsymmetric factorization.

a) If the norms are transposition invariant, that is, the conjugate pairs $\left(r, r_{1}\right)=\left(p_{1}, p\right)=(r, p)$, then

$$
\mathbf{X}=\sum_{\alpha=1}^{\mathbf{k}} \mathbf{a}_{\alpha} \mathbf{a}_{\alpha}^{\prime} / \lambda_{\alpha},
$$

for the geometric structure defined on the rows of $\mathbf{X}$ is identical to the geometric structure defined on the columns of $\mathrm{X}$.

b) If the norms are not transposition invariant, that is, the conjugate pairs $\left(r, r_{1}\right) \neq\left(p_{1}, p\right)$, then

$$
\mathbf{X}=\sum_{\alpha=1}^{\mathbf{k}} \mathbf{a}_{\alpha} \mathbf{b}_{\alpha}^{\prime} / \lambda_{\alpha},
$$

for the geometric structure defined on the rows of $\mathbf{X}$ is different from the geometric structure defined on the columns of $\mathbf{X}$. 


\subsection{A review}

Here, we review published discussed cases in the statistical literature.

a) The centroid decomposition based on $\|\mathbf{A}\|_{\infty \rightarrow 2}=\left\|\mathbf{A}^{\prime}\right\|_{2 \rightarrow 1}$; its transition formulas are

$$
\mathbf{A} \mathbf{u}_{1}=\mathbf{a}_{1} \text { and } \mathbf{v}_{1}=\mathbf{a}_{1} / \sqrt{\mathbf{a}_{1}^{\prime} \mathbf{a}_{1}}
$$

and

$$
\mathbf{A}^{\prime} \mathbf{v}_{1}=\mathbf{b}_{1} \text { and } \mathbf{u}_{1}=\operatorname{sgn}\left(\mathbf{b}_{1}\right)
$$

it is the oldest to our knowledge. First used by Burt (1917), then by Thurstone (1931) to factorize covariance matrices, and used extensively in the psychometric literature before the advent of the computers, see for instance Thurstone (1947), Horst (1965) and Harman (1967). Burt-Thurstone formulation was based on the following criterion:

$$
\max \mathbf{u}^{\prime} \mathbf{A}^{\prime} \mathbf{A} \mathbf{u} \text { subject to } \mathbf{u} \in\{-1,+1\}^{n} \text {; }
$$

its relationship with the matrix norm formulation was shown by Choulakian (2003). In different, but related contexts, it is discussed by Galpin and Hawkins (1987), Chu and Funderlic (2002), Choulakian (2005b), Kwak (2008), McCoy and Tropp (2011). Further, Choulakian (2012) considered it as a particular MAXBET procedure which takes into account the block structure of the variables.

b) $\|\mathbf{A}\|_{1 \rightarrow 1}=\left\|\mathbf{A}^{\prime}\right\|_{\infty \rightarrow \infty}$ is used by Galpin and Hawkins (1987); its transition formulas are

$$
\mathbf{A} \mathbf{u}_{1}=\mathbf{a}_{1} \text { and } \mathbf{v}_{1}=\operatorname{sgn}\left(\mathbf{a}_{1}\right)
$$

and

$$
\mathbf{A}^{\prime} \mathbf{v}_{1}=\mathbf{b}_{1} \text { and } \mathbf{u}_{1}=\mathbf{e}_{\alpha} \text { such that } \alpha=\arg \max _{j}\left|b_{1 j}\right|=\arg \max _{j}|| \mathbf{A}_{* j} \| .
$$

c) The taxicab decomposition is based on $\|\mathbf{A}\|_{\infty \rightarrow 1}=\left\|\mathbf{A}^{\prime}\right\|_{\infty \rightarrow 1}$; its transition formulas are

$$
\mathbf{A} \mathbf{u}_{1}=\mathbf{a}_{1} \text { and } \mathbf{v}_{1}=\operatorname{sgn}\left(\mathbf{a}_{1}\right)
$$

and

$$
\mathbf{A}^{\prime} \mathbf{v}_{1}=\mathbf{b}_{1} \text { and } \mathbf{u}_{1}=\operatorname{sgn}\left(\mathbf{b}_{1}\right) \text {. }
$$

It is the most robust among all the transposition invariant induced norms considered in this paper, and is used extensively by Choulakian and coworkers in developing taxicab correspondence analysis: Choulakian (2004, 2006a, 2008a, 2008b, 2013, 2014), Choulakian et al. (2006, 2013a, 2013b, 2014). We also note that the taxicab decomposition of a covariance matrix is equivalent to the centroid decomposition of the centred dataset. The taxicab norm was first considered by Grothendieck, see the interesting story of the Grothhendieck theorem and its many versions by Pisier (2012). Here, we cite this remarkable result

Grothendieck Inequality: Let $\mathbf{A}=\left(a_{i j}\right)$ be a real matrix of size $m \times n$; then for $i=1, . ., m$ and $j=1, \ldots, n$

$$
\begin{aligned}
\|\mathbf{A}\|_{\infty \rightarrow 1} & =\max _{s_{i}, t_{j}} \sum_{i=1}^{m} \sum_{j=1}^{n} a_{i j} s_{i} t_{j} \text { subject to }\left(s_{i}, t_{j}\right) \in\{-1,1\}^{2} \\
& =\max _{s_{i}, t_{j}} \sum_{i=1}^{m} \sum_{j=1}^{n} a_{i j}<s_{i}, t_{j}>\text { subject to }\left(s_{i}, t_{j}\right) \in S_{2}^{1} \times S_{2}^{1} \\
& \leq K_{d} \max _{\mathbf{s}_{i}, \mathbf{t}_{j}} \sum_{i=1}^{m} \sum_{j=1}^{n} a_{i j}<\mathbf{s}_{i}, \mathbf{t}_{j}>\operatorname{subject} \text { to }\left(\mathbf{s}_{i}, \mathbf{t}_{j}\right) \in S_{2}^{d} \times S_{2}^{d},
\end{aligned}
$$

where $K_{d}$ is the smallest universal constant that depends on $d$ for $d=2,3, \ldots$, but does not depend on $m$ and $n$. By defining $K_{1}=1$, we see that $K_{d} \geq K_{d-1}$ for $d=2,3, \ldots$. The open problem is that there exists a universal constant 
$K_{G}$ such that

$$
K_{G}=\inf _{d} K_{d} \text { such that the inequality in (13) is true. }
$$

It is conjectured that

$$
1.67695 \leq K_{G} \leq \frac{\pi}{2 \log (1+\sqrt{2})}=1.78221 .
$$

An elementary proof of the inequality is given by Blei (1987) or Jameson (1987). A randomization algorithm to compute $\|\mathbf{A}\|_{\infty \rightarrow 1}$ via the Grothendieck inequality is studied by Alon and Naor (2006), and a similar randomization algorithm is used by McCoy and Tropp (2011) to compute (12). Rohn (2000) shows that the computation of $\|\mathbf{A}\|_{\infty \rightarrow 1}$ is NP-hard.

d) The singular value decomposition, SVD, is the standard decomposition, the most used and studied; it is based on $\|\mathbf{A}\|_{2 \rightarrow 2}=\left\|\mathbf{A}^{\prime}\right\|_{2 \rightarrow 2}$, see Horn and Johnson (1990) and Golub and Van Loan (1996). Its transition formulas are

$$
\mathbf{A} \mathbf{u}_{1}=\mathbf{a}_{1} \text { and } \mathbf{v}_{1}=\mathbf{a}_{1} / \sqrt{\mathbf{a}_{1}^{\prime} \mathbf{a}_{1}}
$$

and

$$
\mathbf{A}^{\prime} \mathbf{v}_{1}=\mathbf{b}_{1} \text { and } \mathbf{u}_{1}=\mathbf{b}_{1} / \sqrt{\mathbf{b}_{1}^{\prime} \mathbf{b}_{1}} \text {. }
$$

Example 2: Let us compute a few decompositions to the following matrix

$$
\mathbf{X}=\left(\begin{array}{cc}
1 & -2 \\
-2 & 4 \\
0 & 2
\end{array}\right)
$$

a) Taxicab decomposition: $\|\mathbf{X}\|_{\infty \rightarrow 1}$ is attained at one of the axes: $\mathbf{u}^{\prime}=\left(\begin{array}{ll}1 & 1\end{array}\right)$ or $(1-1)$. For $\mathbf{u}^{\prime}=\left(\begin{array}{ll}1 & 1\end{array}\right)$, $(\mathbf{X u})^{\prime}=\left(\begin{array}{lll}-1 & 2 & 2\end{array}\right)$, and $\|\mathbf{X} \mathbf{u}\|_{\mathbf{1}}=\mathbf{5} \mathbf{u}^{\prime}=\left(\begin{array}{ll}1 & -1\end{array}\right),(\mathbf{X u})^{\prime}=\left(\begin{array}{lll}3 & -6 & -2\end{array}\right)$, and $\|\mathbf{X u}\|_{\mathbf{1}}=\mathbf{1 1}$ So, $\mathbf{u}_{1}^{\prime}=\left(\begin{array}{ll}1 & -1\end{array}\right)$, $\mathbf{a}_{1}^{\prime}=\left(\begin{array}{lll}3 & -6 & -2\end{array}\right), \mathbf{v}_{1}^{\prime}=\operatorname{sgn}\left(\mathbf{a}_{1}^{\prime}\right)=\left(\begin{array}{lll}1 & -1 & -1\end{array}\right), \mathbf{b}_{1}^{\prime}=\left(\mathbf{X}^{\prime} \mathbf{v}_{1}\right)^{\prime}=\left(\begin{array}{ll}3 & -8\end{array}\right), \lambda_{1}=11=\left\|\mathbf{a}_{1}\right\|_{1}=\mathbf{a}_{1}^{\prime} \mathbf{v}_{1}=$ $\left\|\mathbf{b}_{1}\right\|_{1}=\mathbf{b}_{1}^{\prime} \mathbf{u}_{1}$. Note that $\mathbf{u}_{1}=\operatorname{sgn}\left(\mathbf{b}_{1}\right)$. Now the residual matrix, $\mathbf{X}^{(1)}=\mathbf{X}-\mathbf{a}_{1} \mathbf{b}_{1}^{\prime} / \lambda_{1}$, is

$$
\mathbf{X}^{(1)}=\left(\begin{array}{cc}
2 & 2 \\
-4 & -4 \\
6 & 6
\end{array}\right) / 11,
$$

which is of rank 1 . Repeating the above calculations on $\mathbf{X}^{(1)}$, we find $\mathbf{u}_{2}^{\prime}=\left(\begin{array}{ll}1 & 1\end{array}\right), \mathbf{a}_{2}^{\prime}=\left(\begin{array}{lll}1 & -2 & 3\end{array}\right) 4 / 11$, $\mathbf{v}_{2}^{\prime}=\operatorname{sgn}\left(\mathbf{a}_{2}^{\prime}\right)=\left(\begin{array}{lll}1 & -1 & 1\end{array}\right), \mathbf{b}_{2}^{\prime}=\left(\mathbf{X}^{\prime} \mathbf{v}_{2}\right)^{\prime}=\left(\begin{array}{ll}1 & 1\end{array}\right) 12 / 11, \lambda_{2}=24 / 11=\left\|\mathbf{a}_{2}\right\|_{1}=\mathbf{a}_{2}^{\prime} \mathbf{v}_{2}=\left\|\mathbf{b}_{2}\right\|_{1}=\mathbf{b}_{2}^{\prime} \mathbf{u}_{2}$. Note that $\mathbf{u}_{2}=\operatorname{sgn}\left(\mathbf{b}_{2}\right)$. Now the residual matrix, $\mathbf{X}^{(2)}=\mathbf{X}^{(1)}-\mathbf{a}_{2} \mathbf{b}_{2}^{\prime} / \lambda_{2}=\mathbf{0}$. So we have the following decomposition

$$
\mathbf{X}=\left(\begin{array}{lll}
3 & -6 & -2
\end{array}\right)^{\prime}\left(\begin{array}{ll}
3 & -8
\end{array}\right) / 11+\left(\begin{array}{lll}
1 & -2 & 3
\end{array}\right)^{\prime}\left(\begin{array}{ll}
1 & 1
\end{array}\right) 2 / 11 .
$$

b) Centroid decomposition: $\|\mathbf{X}\|_{\infty \rightarrow 2}$ is attained at one of the axes: $\mathbf{u}^{\prime}=\left(\begin{array}{ll}1 & 1\end{array}\right)$ or $\left(\begin{array}{ll}1 & -1\end{array}\right)$. For $\mathbf{u}^{\prime}=\left(\begin{array}{ll}1 & 1\end{array}\right)$, $(\mathbf{X u})^{\prime}=\left(\begin{array}{lll}-1 & 2 & 2\end{array}\right)$, and $\left.\|\mathbf{X} \mathbf{u}\|_{\mathbf{2}}=\sqrt{(\mathbf{9}}\right)$ For $\mathbf{u}^{\prime}=\left(\begin{array}{ll}1 & -1\end{array}\right),(\mathbf{X u})^{\prime}=\left(\begin{array}{lll}3 & -6 & -2\end{array}\right)$, and $\|\mathbf{X u}\|_{\mathbf{2}}=\mathbf{7}$ So, $\mathbf{u}_{1}^{\prime}=\left(\begin{array}{ll}1 & -1\end{array}\right), \mathbf{a}_{1}^{\prime}=\left(\begin{array}{lll}3 & -6 & -2\end{array}\right), \mathbf{v}_{1}^{\prime}=\mathbf{a}_{1}^{\prime} / \lambda_{1}=\left(\begin{array}{lll}3 & -6 & -2\end{array}\right) / 7, \mathbf{b}_{1}^{\prime}=\left(\mathbf{X}^{\prime} \mathbf{v}_{1}\right)^{\prime}=\left(\begin{array}{ll}15 & -34\end{array}\right) / 7, \lambda_{1}=7=$ $\left\|\mathbf{a}_{1}\right\|_{2}=\mathbf{a}_{1}^{\prime} \mathbf{v}_{1}=\left\|\mathbf{b}_{1}\right\|_{1}=\mathbf{b}_{1}^{\prime} \mathbf{u}_{1}$. Note that $\mathbf{u}_{1}=\operatorname{sgn}\left(\mathbf{b}_{1}\right)$. Now the residual matrix, $\mathbf{X}^{(1)}=\mathbf{X}-\mathbf{a}_{1} \mathbf{b}_{1}^{\prime} / \lambda_{1}$, is

$$
\mathbf{X}^{(1)}=\left(\begin{array}{cc}
4 & 4 \\
-8 & -8 \\
30 & 30
\end{array}\right) / 49
$$

which is of rank 1. Repeating the above calculations on $\mathbf{X}^{(1)}$, we find $\mathbf{u}_{2}^{\prime}=\left(\begin{array}{ll}1 & 1\end{array}\right), \mathbf{a}_{2}^{\prime}=\left(\begin{array}{lll}2 & -4 & 15\end{array}\right) 4 / 49$, $\mathbf{v}_{2}^{\prime}=\mathbf{a}_{2}^{\prime} /\left\|\mathbf{a}_{2}\right\|_{2}=\left(\begin{array}{lll}2 & -4 & 15\end{array}\right) /(7 \sqrt{5}), \mathbf{b}_{2}^{\prime}=\left(\mathbf{X}^{\prime} \mathbf{v}_{2}\right)^{\prime}=\left(\begin{array}{ll}1 & 1\end{array}\right) 2 \sqrt{5} / 7, \lambda_{2}=4 \sqrt{5} / 7=\left\|\mathbf{a}_{2}\right\|_{2}=\mathbf{a}_{2}^{\prime} \mathbf{v}_{2}=\left\|\mathbf{b}_{2}\right\|_{1}=$ $\mathbf{b}_{2}^{\prime} \mathbf{u}_{2}$. Note that $\mathbf{u}_{2}=\operatorname{sgn}\left(\mathbf{b}_{2}\right)$. Now the residual matrix, $\mathbf{X}^{(2)}=\mathbf{X}^{(1)}-\mathbf{a}_{2} \mathbf{b}_{2}^{\prime} / \lambda_{2}=\mathbf{0}$. So we have the following 
decomposition

$$
\mathbf{X}=\left(\begin{array}{lll}
3 & -6 & -2
\end{array}\right)^{\prime}\left(\begin{array}{lll}
15 & -34
\end{array}\right) / 49+\left(\begin{array}{lll}
2 & -4 & 15
\end{array}\right)^{\prime}\left(\begin{array}{ll}
1 & 1
\end{array}\right) 2 / 49 .
$$

c) Extreme decomposition: $\|\mathbf{X}\|_{1 \rightarrow \infty}$ is attained on one of the canonical basis vectors: $\mathbf{e}_{1}^{\prime}=\left(\begin{array}{ll}1 & 0\end{array}\right)$ or $\mathbf{e}_{2}^{\prime}=\left(\begin{array}{ll}0 & 1\end{array}\right)$. For $\mathbf{u}=\mathbf{e}_{1},(\mathbf{X u})^{\prime}=\left(\begin{array}{lll}1 & -2 & 0\end{array}\right)$, and $\|\mathbf{X u}\|_{\infty}=\mathbf{2}$ For $\mathbf{u}=\mathbf{e}_{2},(\mathbf{X u})^{\prime}=\left(\begin{array}{lll}-2 & 4 & 2\end{array}\right)$, and $\|\mathbf{X u}\|_{\infty}=\mathbf{4}$ So, $\mathbf{u}_{1}=\mathbf{e}_{2}, \mathbf{a}_{1}=\mathbf{X}_{* 2}$ the second column of $\mathbf{X}, \mathbf{v}_{1}^{\prime}=\left(\begin{array}{lll}0 & 1 & 0\end{array}\right), \mathbf{b}_{1}^{\prime}=\left(\mathbf{X}^{\prime} \mathbf{v}_{1}\right)^{\prime}=\left(\begin{array}{ll}-2 & 4\end{array}\right), \lambda_{1}=4=\left\|\mathbf{a}_{1}\right\|_{\infty}=$ $\mathbf{a}_{1}^{\prime} \mathbf{v}_{1}=\left\|\mathbf{b}_{1}\right\|_{\infty}=\mathbf{b}_{1}^{\prime} \mathbf{u}_{1}$. Note that $\mathbf{u}_{1}=\mathbf{e}_{2} \operatorname{sgn}\left(b_{12}\right)$. Now the residual matrix, $\mathbf{X}^{(1)}=\mathbf{X}-\mathbf{a}_{1} \mathbf{b}_{1}^{\prime} / \lambda_{1}$, is

$$
\mathbf{X}^{(1)}=\left(\begin{array}{ll}
0 & 0 \\
0 & 0 \\
1 & 0
\end{array}\right) / 11,
$$

which is of rank 1. Repeating the above calculations on $\mathbf{X}^{(1)}$, we find $\mathbf{u}_{2}=\mathbf{e}_{1}, \mathbf{a}_{2}^{\prime}=\left(\begin{array}{lll}0 & 0 & 1\end{array}\right), \mathbf{v}_{2}=\mathbf{a}_{2}$, $\mathbf{b}_{2}^{\prime}=\left(\mathbf{X}^{\prime} \mathbf{v}_{2}\right)^{\prime}=\left(\begin{array}{ll}1 & 0\end{array}\right), \lambda_{2}=1=\left\|\mathbf{a}_{2}\right\|_{\infty}=\mathbf{a}_{2}^{\prime} \mathbf{v}_{2}=\left\|\mathbf{b}_{2}\right\|_{\infty}=\mathbf{b}_{2}^{\prime} \mathbf{u}_{2}$. Note that $\mathbf{u}_{2}=\mathbf{e}_{1} \operatorname{sgn}\left(b_{21}\right)$. Now the residual matrix, $\mathbf{X}^{(2)}=\mathbf{X}^{(1)}-\mathbf{a}_{2} \mathbf{b}_{2}^{\prime} / \lambda_{2}=\mathbf{0}$. So we have the following decomposition

$$
\mathbf{X}=\left(\begin{array}{lll}
-2 & 4 & 2
\end{array}\right)^{\prime}\left(\begin{array}{ll}
-2 & 4
\end{array}\right) / 4+\left(\begin{array}{lll}
0 & 0 & 1
\end{array}\right)^{\prime}\left(\begin{array}{ll}
1 & 0
\end{array}\right) .
$$

d) Singular value decomposition: It is based on the singular values and singular vectors of $\mathbf{X}: \lambda_{1}=5.3191$, $\mathbf{v}_{1}^{\prime}=\left(\begin{array}{lll}-0.4197 & 0.8393 & 0.3455\end{array}\right), \mathbf{a}_{1}=\lambda_{1} \mathbf{v}_{1}, \mathbf{u}_{1}=\left(\begin{array}{lll}-0.3945 & 0.9189\end{array}\right)$ and $\mathbf{b}_{1}=\lambda_{1} \mathbf{u}_{1} ; \lambda_{2}=0.8408, \mathbf{v}_{2}^{\prime}=$ $\left(\begin{array}{lll}-0.1545 & 0.3090-0.9384\end{array}\right), \mathbf{a}_{2}=\lambda_{2} \mathbf{v}_{2}, \mathbf{u}_{2}=\left(\begin{array}{lll}-0.9189 & -0.3945\end{array}\right)$ and $\mathbf{b}_{2}=\lambda_{2} \mathbf{u}_{2}$. So we have the following decomposition

$$
\begin{aligned}
& \mathbf{X}=\left(\begin{array}{llll}
-0.4197 & 0.8393 & 0.3455
\end{array}\right)^{\prime}(-0.39450 .9189) 5.3191+ \\
& \left(\begin{array}{llll}
-0.1545 & 0.3090 & -0.9384
\end{array}\right)^{\prime}(-0.9189-0.3945) 0.8408 \text {. }
\end{aligned}
$$

\section{Remark 2:}

a) We note that the factors $\left(\mathbf{a}_{\alpha}, \mathbf{b}_{\alpha}\right)$ are determined up to proportionality, and the four decompositions in equations (15) through (18) of the data set X given in (14) are essentially different. This is a much discussed and important topic, named factor indeterminacy problem; see for instance Mulaik (1987). We can recast or reformulate the factor indeterminacy problem within a geometric setting: If $\operatorname{Rank}(\mathbf{X}) \geq 2$, then there are infinite number of different factorizations depending on the values of $r \geq 1$ and $p \geq 1$ for $\mathbf{X} \in B\left(l_{r}^{n}, l_{p}^{m}\right)$.

b) The decomposition of $\mathbf{X}$ is essentially unique (up to proportionality) if and only if $\operatorname{rank}(\mathbf{X})=1$.

c) Conditions for essential uniqueness of decompositions for three-way arrays or tensors is an active area of research; and Kruskal's sufficiency theorem is the most famous general result, see Rhodes (2010). For an overview of the literature on tensor decomposition, see the interesting review by Ten Berge (2011); for some computational numerical results concerning the typical ranks of three-way arrays over the real field, see Choulakian (2010).

\subsection{A family of Euclidean multidimensional scaling models}

Let $\boldsymbol{\Delta}=\left(\delta_{i j}\right)$ be a symmetric $n \times n$ matrix with nonnegative elements and zeros on the diagonal, representing the dissimilarities of $n$ objects. The aim of multidimensional scaling (MDS) techniques is to find a configuration of the $n$ points, which best match the original dissimilarities as much as possible. We shall consider the framework of the classical MDS, named also principle coordinate analysis, where we suppose that the dissimilarities represent Euclidean distances, which implies that there exists a set of $n$ centered points in a Euclidean space, denoted by $\left\{\mathbf{f}_{i}: i=1, \ldots, n\right\}$, such that

$$
\delta_{i j}^{2}=\left\|\mathbf{f}_{i}-\mathbf{f}_{j}\right\|_{2}^{2} .
$$

We thus have the following well known relationship

$$
\begin{aligned}
\mathbf{Q} & =-\frac{1}{2}\left(\mathbf{H} \boldsymbol{\Delta}^{*} \mathbf{H}\right) \\
& =\mathbf{F}^{\prime} \mathbf{F}
\end{aligned}
$$


where, $\boldsymbol{\Delta}^{*}=\left(\delta_{i j}^{2}\right), \mathbf{F}=\left[\mathbf{f}_{1}, \ldots, \mathbf{f}_{n}\right]$ and $\mathbf{H}=\mathbf{I}_{n}-\mathbf{1}_{n} \mathbf{1}_{n}^{\prime} / n$ is the centering matrix, $\mathbf{I}_{n}$ the identity matrix and $\mathbf{1}_{n}$ the vector of ones. So the matrix $\mathbf{Q}$ is positive semi-definite, and it is equal to $\mathbf{F}^{\prime} \mathbf{F}$, where $\mathbf{F}$ is unknown. Now suppose that $\mathbf{F} \in B\left(l_{r}^{n}, l_{p}^{m}\right)$. Then

$$
\|\mathbf{F}\|_{r \rightarrow p}=\max \left\{\|\mathbf{F u}\|_{p}: \mathbf{u} \in S_{r}^{n}\right\}
$$

can be expressed as a linear function of $\mathbf{Q}$ if $p=2$ and $r \geq 1$; that is

$$
\begin{aligned}
\|\mathbf{F}\|_{r \rightarrow 2}^{2} & =\mathbf{u}^{\prime} \mathbf{F}^{\prime} \mathbf{F u} \quad \text { subject to } \mathbf{u} \in S_{r}^{n} \\
& =\mathbf{u}^{\prime} \mathbf{Q u} \quad \text { subject to } \mathbf{u} \in S_{r}^{n} .
\end{aligned}
$$

Factorizing $\mathbf{Q}$ into $\mathbf{F}^{\prime} \mathbf{F}$ by (19), we obtain a family of Euclidean multidimensional scaling (MDS) models as a function of $r \geq 1$. Three particular cases are worthy of mention:

a) For $r=2$, we obtain the classical MDS, see for instance Torgerson (1952) and Gower (1966). Each $\mathbf{f}_{\alpha}$ is an eigenvector of $\mathbf{Q}$; see also Proposition 3.

b) For $r=\infty$, we get the centroid MDS, where we maximize the Burt-Thurstone criterion (11), see also Proposition 1.

c) For $r=1$, we get the dominant MDS; its computation is extremely simple and fast, see Proposition 2.

Example 3: We consider the Facial Expressions data found in Borg and Groenen (2005, p. 76) of dimension $13 \times 13$, where $n=13$ is the number of person's facial expressions. The aim of the study is the correct identification of intended emotional message from a person's facial expression. Furthermore, Table 4.3, p. 75 in Borg and Groenen, provide Schlosberg empirical scale values that classify the facial expressions into three classes: pleasantunpleasant (PU), attention-rejection (AR) and tension-sleep (TS). Borg and Groenen (2005, subsection 4.3) found that the first two dimensions of ordinal MDS reproduced quite accurately the three classes: the first dimension representing PU and the second dimension representing AR and TS, because the correlations between the first two calculated dimensions and the Schlosberg empirical scale values are quite high for ordinal MDS. Table 1 compares the correlation values obtained by four MDS approaches; the ordinal MDS correlation values are reproduced from Borg and Groenen (2005, p.77, Table 4.6): The centroid MDS produced results as good as the ordinal MDS.

Table 1: Facial Expressions Data: Correlation values.

\begin{tabular}{|l||l|l|l|}
\hline MDS & corr(DIM1,PU) & corr(DIM2,AR) & corr(DIM2,TS) \\
\hline ordinal & 0.94 & 0.86 & 0.87 \\
\hline classical & 0.91 & 0.80 & 0.83 \\
\hline centroid & 0.93 & 0.86 & 0.89 \\
\hline dominant & 0.91 & 0.78 & 0.70 \\
\hline
\end{tabular}

\section{The French school of data analysis}

Benzécri (1973a), who was a pure mathematician in geometry in the 1950s, is considered the father of the french school of data analysis; he developed a geometric generalized Euclidean framework for multidimensional data analysis by introducing two metric matrices (square and positive definite) $\mathbf{M}$ and $\mathbf{N}$, thus defining two Euclidean quadratic normed spaces $l_{2}^{n}(\mathbf{N})$ et $l_{2}^{m}(\mathbf{M})$.

Definition: Let $l_{2}^{n}(\mathbf{N}):=\left(\mathbb{R}^{n},\|\cdot\|_{\mathbf{N}}\right)$, where we define the following scalar product

for $\mathbf{x} \in \mathbb{R}^{n}$ and $\mathbf{y} \in \mathbb{R}^{n},<\mathbf{y}, \mathbf{x}>_{\mathbf{N}}=\mathbf{y}^{\prime} \mathbf{N} \mathbf{x}$;

and the quadratic norm based on it

for $\mathbf{x} \in \mathbb{R}^{n},\|\mathbf{x}\|_{\mathbf{N}}=\left(\mathbf{x}^{\prime} \mathbf{N} \mathbf{x}\right)^{1 / 2}$.

The $\|\mathbf{x}\|_{\mathbf{N}}$ represents a generalized distance of the vector $\mathbf{x} \in \mathbb{R}^{n}$ with respect to the origin, and it satisfies the four properties of a norm, N1 to N4 listed in section 2.

We define: a) The unit sphere to be

$$
S_{p}^{n}(\mathbf{N})=\left\{\mathbf{x} \in \mathbb{R}^{n}:\|\mathbf{x}\|_{N}=1\right\} .
$$

Stat., Optim. Inf. Comput. Vol. 4, March 2016 
b) The normed functional of $\mathrm{x} \in \mathbb{R}^{n}$ to be

$$
\varphi_{\mathbf{N}}(\mathbf{x})=\mathbf{N} \mathbf{x} /\|\mathbf{x}\|_{\mathbf{N}} \in S_{2}^{n}(\mathbf{N})^{*}
$$

for

$$
\begin{aligned}
\|\mathbf{x}\|_{\mathbf{N}} & =\sqrt{\langle\mathbf{x}, \mathbf{x}\rangle_{\mathbf{N}}} \\
& =\left\langle\varphi_{\mathbf{N}}(\mathbf{x}), \mathbf{x}>.\right.
\end{aligned}
$$

Note that, for the particular value of $\mathbf{N}=\mathbf{I}_{n}, \varphi_{\mathbf{N}}(\mathbf{x})=\varphi_{\mathbf{2}}(\mathbf{x})$, and the framework of the Euclidean quadratic normed space $l_{2}^{n}(\mathbf{N})$, becomes equivalent to the framework of the ordinary Euclidean normed space $l_{2}^{n}=l_{2}^{n}(\mathbf{I})$; thus for $\mathbf{A} \in B\left(l_{2}^{n}(\mathbf{N}), l_{2}^{m}(\mathbf{M})\right)$ its duality diagram and its transition formulas can be obtained by replacing $\varphi_{\mathbf{2}}(\mathbf{x})$ by $\varphi_{\mathbf{N}}(\mathbf{x})$ in the duality diagram described in section 2 . That is, for $\mathbf{A} \in B\left(l_{2}^{n}(\mathbf{N}), l_{2}^{m}(\mathbf{M})\right)$ its duality diagram is

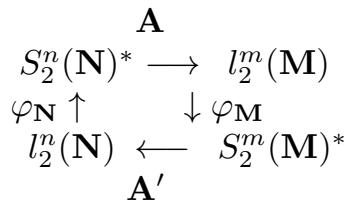

which represents the following transition formulas

$$
\mathbf{A} \mathbf{u}_{1}=\mathbf{a}_{1} \text { and } \mathbf{v}_{1}=\varphi_{\mathbf{M}}\left(\mathbf{a}_{1}\right)=\mathbf{M} \mathbf{a}_{1} /\left\|\mathbf{a}_{1}\right\|_{\mathbf{M}}=\mathbf{M a} \mathbf{a}_{1} / \lambda_{1}
$$

and

$$
\mathbf{A}^{\prime} \mathbf{v}_{1}=\mathbf{b}_{1} \text { and } \mathbf{u}_{1}=\varphi_{\mathbf{N}}\left(\mathbf{b}_{1}\right)=\mathbf{N b}_{1} /\left\|\mathbf{b}_{1}\right\|_{\mathbf{N}}=\mathbf{N b}_{1} / \lambda_{1} .
$$

The solution of the last two equations can be reexpressed as a generalized eigenvalue-eigenvector problem in the following way: From the last two equations we get

$$
\begin{aligned}
\mathbf{a}_{1} & =\mathbf{A u}_{1} \\
& =\mathbf{A N b}_{1} / \lambda_{1} \\
\mathbf{M}^{-1} \mathbf{v}_{1} \lambda_{1} & =\mathbf{A N A}^{\prime} \mathbf{v}_{1} / \lambda_{1}
\end{aligned}
$$

from which one gets

$$
\operatorname{MANA}^{\prime} \mathbf{v}_{1}=\lambda_{1}^{2} \mathbf{v}_{1}
$$

and similarly

$$
\mathbf{N A} \mathbf{M A}_{1}=\lambda_{1}^{2} \mathbf{u}_{1}
$$

In the last two equations the eigenequations are functions of principal axes $\mathbf{u}_{1}$ and $\mathbf{v}_{1}$. However, one can reexpress them as functions of projected factor scores

$$
\mathbf{A N A}^{\prime} \mathbf{M a}_{1}=\lambda_{1}^{2} \mathbf{a}_{1}
$$

and similarly

$$
\mathbf{A}^{\prime} \mathbf{M A N \mathbf { b } _ { 1 }}=\lambda_{1}^{2} \mathbf{b}_{1}
$$




\section{Conclusion}

We embedded the ordinary SVD into a larger family based on induced matrix norms, and provided the transition formulas and a simple criss-cross iterative procedure to compute the principal axes and principal factor scores. Given that there are infinite number of SVD like decompositions, depending on the underlying induced norms, one is tempted to ask which is the best?

It is quite ironic that the centroid decomposition, the oldest method, was recently rediscovered and restudied as a robust method, see Choulakian (2005b), Kwak (2008), McCoy and Tropp (2011), after being dumped almost sixty years ago for the following reason given in Hubert et al. (2000, p.76) "Comments in Guttman (1944) and elsewhere (e.g., Horst (1965) and Harman (1967)) with regard to this centroid strategy generally considered it a poor approximation to what could be generated from Hotelling's method that would choose successive unit length vectors to produce a rank reduction by identifying (through an iterative strategy) the eigenvector associated with the largest eigenvalue for each of the residual matrices successively obtained. At the time, however, the centroid method was computationally much less demanding than Hotelling's iterative (or power) method for obtaining each of the principal components (again, one-at-a-time and reducing rank at each iteration); for this reason alone, the centroid method was a very common factorization strategy until electronic computing capabilities became more widely available". This comment shows that the centroid method was a victim of the habit of using mathematical methods in statistics based on optimal criteria, as if optimality is a guarantee of efficiency.

The arguments advanced by Benzécri on the advantages of the Euclidean geometry over the taxicab geometry for multidimensional data analysis are both computational and metaphysical. On the use of L1 distance in data analysis, Benzécri (1977, page 13) commented in the following way" elle (L1) ne permet pas d'utiliser la géométrie euclidienne multidimensionnelle; elle donnera des résultats qui qualitativement ressembleront à ceux obtenus par la distance...quadratique; mais au prix de calculs plus compliqués et sous une forme moins commode. Sans permettre à l'outil mathématique de défigurer le réel, on doit lui concéder que la transmission à l'esprit humain d'un vaste ensemble de données synthétisé (résumé; rendu perceptible par le calcul) ait ses lois propres. (On se souvient que le primat de la géométrie euclidienne est admis par Torgerson)". The ease of computation argument is very similar to Gauss's argument in the adoption of least squares criterion in the linear regression model. While the metaphysical argument, if my understanding is correct, is that: the transmission to the human spirit of a synthesis of a collection of data has its proper laws, which are based on the Euclidean geometry.

Ten Berge (2005, personal communication) thought that the centroid method produced good results, but it was not mathematically well understood during the last century. Benzécri (1973b, page 1 in Avant-propos) considered data analysis an experimental science, and that he has a predeliction for the case studies in his books. A similar thought is also found in Tukey: "The test of a good procedure is how well it works, not how well it is understood".

As to the question asked which decomposition is the best? Mathematically, the SVD is the best and the reference, but quite sensitive to outlying observations: So, we suggest the joint use of SVD and the taxicab decomposition, or, the SVD and the centroid decomposition. For an example, concerning the joint use of correspondence analysis and taxicab correspondence analysis, where the two methods produced completely different results, see for instance among others, Choulakian et al. (2006) and Gauthier and Choulakian (2015).

Acknowledgement: This research was financed by the Natural Sciences and Engineering Research Council of Canada. The author thanks Bernard Fichet, François Brucker and Pascal Préa of the École Centrale de Marseille for the invitation, for which this paper has been specially prepared.

\section{REFERENCES}

1. Alon, N. and Naor, A. Approximating the cut-norm via Grothendieck's inequality. SIAM Journal on Computing, 35, 787-803, (2006).

2. Benzécri, J.P. (1973a). L’Analyse des Données: Vol. 2: L’Analyse des Correspondances. Paris: Dunod.

3. Benzécri, J.P. (1973b). L’Analyse des Données: Vol. 1: La Taxinomie. Paris: Dunod.

4. Benzécri, J.P. (1977). Histoire et Préhistoire de l'Analyse des Données: V: L'Analyse des correspondances. Les Cahiers de l'Analyse des Données, II(1), 9-53. 
5. Blei, R.C. (1987). An elementary proof of the Grothendieck inequality. Proceedings of the American Mathematical Society, 100(1), $58-60$.

6. Borg, I. and Groenen, P.G. (2005). Modern Multidimensional Scaling. 2nd edition, NY: Springer Verlag.

7. Boyd, D.W. (1974). The power method for $l_{p}$ norms. Linear Algebra and its Applications, 9, 95-101.

8. Burt, C. (1917). The Distribution and Relations of Educational Abilities. London, U.K: P.S. King \& son.

9. Choulakian, V. (2003). The optimality of the centroid method. Psychometrika, 68, 473-475.

10. Choulakian, V. (2004). A comparison of two methods of principal component analysis. In COMPSTAT'2004 edited by J. Antoch,Physica-Verlag/Springer, 793-798.

11. Choulakian, V. (2005a). Transposition invariant principal component analysis in $\mathrm{L}_{1}$ for long tailed data. Statistics and Probability Letters,71, 23-31.

12. Choulakian, V. (2005b). $\mathrm{L}_{1}$-norm projection pursuit based principal component analysis. Computational Statistics and Data Analysis, 50,1441-1451.

13. Choulakian, V. (2006a). Taxicab correspondence analysis. Psychometrika,71, 333-345.

14. Choulakian, V. (2006b). $\mathrm{L}_{1}$ norm projection pursuit principal componentanalysis. Computational Statistics and Data Analysis, 50, 1441-1451.

15. Choulakian, V. (2008a). Taxicab correspondence analysis of contingency tables with one heavyweight column. Psychometrika, 73, 309-319.

16. Choulakian, V. (2008b). Multiple taxicab correspondence analysis. Advances in data Analysis and Classification, 2, 177-206.

17. Choulakian, V. (2010). Some numerical results on the rank of genericthree-way arrays over R. SIAM Journal of Matrix Analysis and Applications, 31(4), 1541-1551

18. Choulakian, V. (2012). Picture of all solutions of successive 2-block MAXBET problems. Psychometrika, 76(4), 550-563.

19. Choulakian V. (2013). The simple sum score statistic in taxicab correspondence analysis. In Advances in Latent Variables (ebook), eds. Brentari E.and Carpita M., Vita e Pensiero, Milan, Italy, ISBN 978883432556 8, 6 pages.

20. Choulakian, V. (2014). Taxicab correspondence analysis of ratings and rankings. Journal de la Société Française de Statistique.155(4), 1-23.

21. Choulakian, V., Allard, J. and Simonetti, B. (2013). Multiple taxicab correspondence analysis of a survey related to health services Journal of Data Science, 11(2), 205-229.

22. Choulakian, V. and de Tibeiro, J. (2013). Graph partitioning by correspondence analysis and taxicab correspondence analysis. Journal of Classification, 30(3), 397-427.

23. Choulakian, V., Simonetti, B. and Gia, T.P. (2014). Some new aspects of taxicab correspondence analysis. Statistical Methods and Applications, 23(3), 401-416.

24. Choulakian, V., Kasparian, S., Miyake, M., Akama, H., Makoshi, N., Nakagawa, M. (2006). A statistical analysis of synoptic gospels. JADT'2006, pp.281-288.

25. Chu, M.T. and Funderlic, R.E. (2002). The centroid decomposition: relationships between discrete variational decompositions and SVDs. SIAM J. Matrix Analysis and Applications, 23, 1025-1044.

26. Chu, M.T., Funderlic, R.E. and Golub, G.H. (1995). A rank-one reduction formula and its applications to matrix factorizations. SIAM Review, 37(4), 512-530.

27. De La Cruz, O. and Holmes, S. (2011). The duality diagram in data analysis:Examples of modern applications. Annals of Applied Statistics, 5(4),2266-2277.

28. Drakakis. K. and Pearlmutter, B.A. (2009). On the calculation of the $l_{2} \rightarrow l_{1}$ induced matrix norm. International Journal of Algebra, 3(5), 231-240.

29. Gabriel, K.R. and Zamir, S. (1979). Lower rank approximation of matrices by least squares with any choice of weights Technometrics, 21, 489-498.

30. Galpin, J.S. and Hawkins, D.M. (1987). $\mathrm{L}_{1}$ estimation of a covariance matrix. Computational Statistics and Data Analysis, 5 , 305-319.

31. Gauthier, S.M. and Choulakian, V. (2015). Taxicab correspondence analysis of abundance data in archeology: three case studies revisited. To appear in Archeologia e Calcolatori, 26.

32. Golub, G.H. and Van Loan, C.F. (1996). Matrix Computations. 3rd edition, John Hopkins Studies in the Mathematical Sciences, The Johns Hopkins University Press, Baltimore, MD, ISBN 978-0-8018-5414-9.

33. Gower, J.C. (1966). Some distance properties of latent root and vector methods in multivariate analysis. Biometrika, 53, 325-338.

34. Guttman, L. (1944). General theory and methods for matric factoring. Psychometrika, 9, 1-16.

35. Harman, H.H. (1967). Modern Factor Analysis. Chicago, IL: The University of Chicago Press.

36. Holmes, S. (2008). Mutivariate analysis: The French way. In Probability and Statistics: Essays in honor of David Freedman, eds. D. Nolan and T. Speed, pp. 219-233. Ohio, USA: Institute of Mathematical Statistics.

37. Horn, R.A. and Johnson, C.R. (1990). Matrix Analysis. Cambridge University Press, NY.

38. Horst, P. (1965). Factor Analysis of Data Matrices. NY: Holt, Rinehart and Winston.

39. Hubert, L., Meulman, J. and Heiser, W. (2000). Two purposes for matrix factorization: A historical appraisal. SIAM Review, 42, 68-82.

40. Jameson, G.J.O. (1987). Summing and Nuclear Norms in Banach Space Theory. London Mathematical Society Student Texts 8, Cambridge University Press, Cambridge.

41. Kreyszig, E. (1978). Introductory Functional Analysis with Applications J. Wiley and Sons, N.Y.

42. Kwak, N. (2008). Principal component analysis based on L1-norm maximization. IEEE Transactions on Pattern Analysis and Machine Intelligence, 30(9), 1672-1680.

43. Lewis, A.D. (2010). A top nine list: Most popular induced matrix norms. Downloaded from http://www.mast.queensu.ca/ andrew/notes/pdf/2010b.pdf

44. McCoy, M. and Tropp, J. (2011). Two proposals for robust PCA usingsemidefinite programming. Electronic Journal of Statistics, $5,1123-1160$ 
45. Mulaik, S. (1987). A brief history of the phlosophical foundations of exploratory factor analysis. Multivariate Behavioral Research, 22, 267-305

46. Pisier, G. (2012). Grothendieck's theorem, past and present. Bulletin of the American Mathematical Society, 49 (2): $237-323$.

47. Rohn, J. (2000). Computing the norm $\|\mathbf{A}\|_{\infty \rightarrow 1}$ is NP-hard. Linear and Multilinear Algebra, 47 (3), 195-204.

48. Rhodes, J.A. (2010). A concise proof of Kruskal's theorem on tensor decomposition. Linear Algebra and its Applications, 432 (7), 1818-1824.

49. Ten Berge, J.M.F. (2011). Simplicity and typical rank results for three-way arrays. Psychometrika, 76(1), 3-12.

50. Thurstone, L.L. (1931). Multiple factor analysis. Psychological Review, 38, 406-427.

51. Thurstone, L.L. (1947). Multiple-Factor Analysis. The University of Chicago Press, Chicago

52. Torgerson, W.S. (1952). Multidimensional scaling: 1. Theory and method. Psychometrika, 17, 401-419.

53. Wold, H. (1966). Estimation of principal components and related models by iterative least squares. In Multivariate Analysis, ed. Krishnaiah,P.R., N.Y: Academic Press, 391-420. 